\title{
Transformation of spectra of refraction index fluctuations in axisymmetric supersonic jet with the increase in the distance from the nozzle
}

\author{
Dmitrii Marakasov ${ }^{1, *}$, Valentina Sazanovich ${ }^{2}$, Ruvim Tsvyk ${ }^{1}$, and Andrei Shesternin ${ }^{1}$ \\ ${ }^{1}$ V.E. Zuev Institute of Atmospheric Optics SB RAS, Wave Propagation Laboratory, 634055 Ac. \\ Zuev square, 1, Tomsk, Russia
}

\begin{abstract}
In this paper the results of reconstruction of the radial dependence of refraction index fluctuations spectra in axisymmetric supersonic jet from laser transillumination data are presented. The reconstruction algorithm uses experimental spectra of laser radiation, received behind the jet, and the distribution of time-averaged axial air velocity within the jet. Laser transillumination experiments were performed on the Vertical Jet Unit of ITAM SB RAS (Novosibirsk, Russia). Reconstruction results show the refraction index spectra obey the power-law model, with power index different from that of the Kolmogorov turbulence model $(-11 / 3)$. Within the first two barrels of the jet the power index is changing from $\sim-1$ near the nozzle to $\sim-2.7$ near the second Mach disk. Also some variations of power index and amplitude coefficient with the distance from the jet axis are found.
\end{abstract}

Currently the most common contact methods of measurement of pulsation characteristics of the flow at wind tunnels have fatal flaws stemming from their very essence. The direct interaction of the sensors with the supersonic flow leads, on the one hand, to their rapid destruction, and on the other hand, to the distortion of flow structure.

The dynamics of fluid or gas flows can also be studied with the use of noncontact optical methods. Among these are speckle photography and speckle interferometry methods [1], in which the source of information is represented by intensity fluctuations of the laser radiation passed through the flow and a diffuse plate. The widely used methods of shadow visualization (Schlieren photography) do not allow the spatial spectrum of refractive index fluctuations to be determined from the obtained images [2]. Shadow images are integral characteristics of the refractive index in the entire radiation propagation path, and the spatial distribution of the refractive index in different flow layers cannot be reconstructed from them.

Hence it is of interest to study the possibilities of remote diagnostics of the dencity inhomogeneities in supersonic gas flow based on turbulent intensity fluctuations of translucent optical radiation, which does not require the presence of scattering particles.

\footnotetext{
*Corresponding author: $\underline{\text { mda@iao.ru }}$
} 

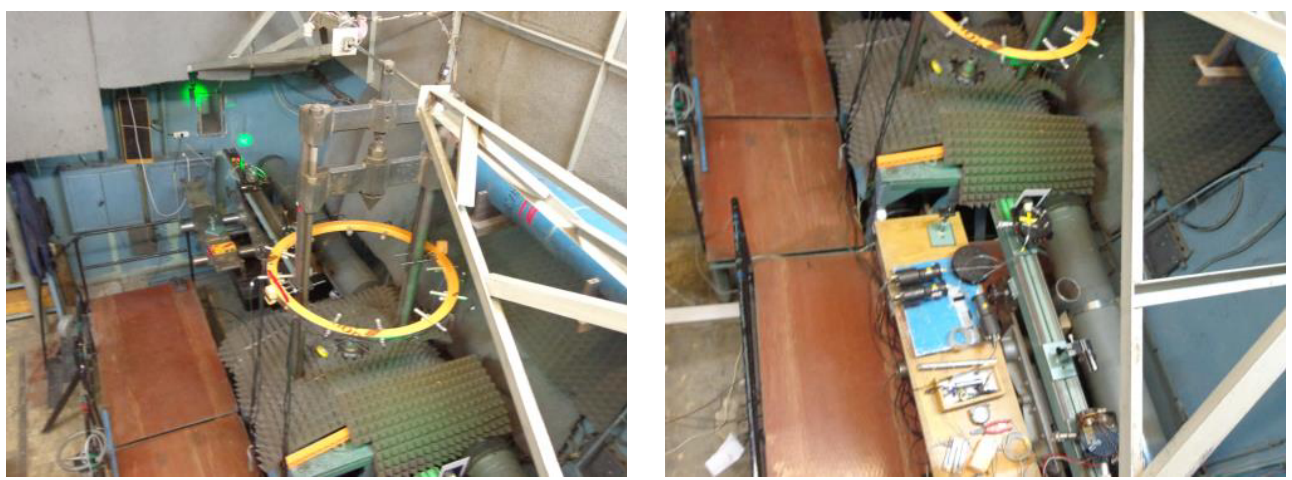

Fig. 1. Vertical jet unit with optical equipment. Source part (right) and receiver part (left).

Based on the algorithm of determining the radial dependence of spatial spectra of the refractive index from time spectra of the received power of laser radiation translucent the axisymmetric jet [3], the transformation of the refraction index spectra accompanied by the change of the air velocity and the turbulence with distance from the nozzle exit was investigated. The original data were obtained in experiments performed in 2016 at the Vertical Jet Unit (VJU) of ITAM SB RAS (Figure 1) also the results of the earlier experiments (2013) were used.

The supersonic jet generated by Witoshinski nozzle with a diameter of $30 \mathrm{~mm}$, went straight up to the room of size $5 \times 5 \times 3 \mathrm{~m}$. On opposite sides of the jet on the optical tables were located laser source and forming diaphragm on one side and the receiving part on the other (Figure 1). The laser beam is converted in a spherical wave by the diaphragm located at a distance of $1 \mathrm{~m}$ from the jet axis. At a distance of $1 \mathrm{~m}$ behind the jet laser radiation perturbed by inhomogeneities inside the jet was falling on the entrances of the three lightguides with a diameter of $0.15 \mathrm{~mm}$, which carried signals to three PMT. The output of photomultipliers was recorded using oscilloscope TiePie Handyscope HS4diff with a sampling rate of $5 \mathrm{MHz}$ per channel. The record length was 130000 counts. The distance between the lightguides was $2 \mathrm{~mm}$, which corresponded to the spacing of the beams inside the jet $1 \mathrm{~mm}$. Optical table with the laser source and forming the diaphragm moved vertically (along the jet axis), the table of the receiving part moved both vertically and horizontally (perpendicularly to the optical axis). This allowed to vary as the distance of the optical axis from the nozzle exit $(42-90 \mathrm{~mm}$ ) and impact parameter (distance from the beam to the axis of the jet at the point of convergence) up to $23 \mathrm{~mm}$.

Reconstruction algorithm for the radial dependence of the spectra [3] uses a priori information about the time-averaged flow velocity in the corresponding cross section of the jet. The axial component of the average velocity was calculated from the results of numerical simulation of a jet with the ratio of the pressure in the settling chamber to atmospheric $n p r=5$ in a package Fluent 6.3. The radial dependences of the axial velocity for some distances from the nozzle exit corresponding to the second barrel are presented in Fig. 2.

It is seen that in the vicinity of the Mach disk the axial velocity in the inner region of the jet is changed in $\sim 5$ times from subsonic values near the axis of the jet to supersonic closely to its external border. In the region between the first and second Mach disks its relative variations over the jet cross section are in the range of $15-20 \%$. Thus, the renormalization of frequencies in the framework of the reconstruction algorithm of the radial dependence of spectra of density fluctuations is essential for sections close to the Mach disk, and does not introduce large refiners for intermediate sections. 


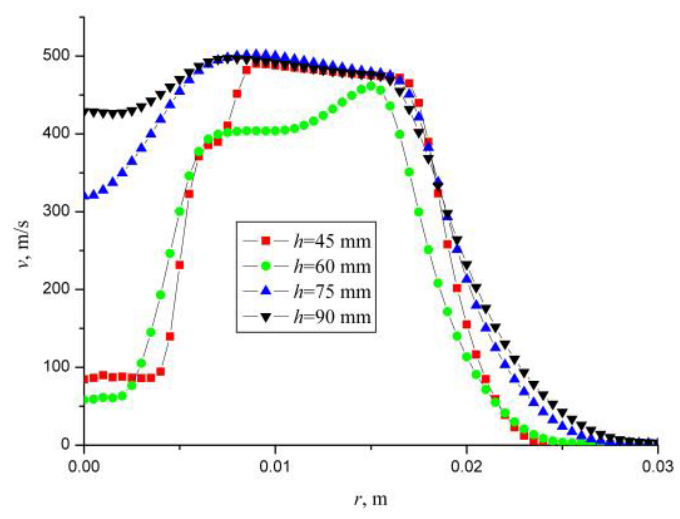

Fig. 2. Axial velocity distribution in the supersonic jet with $n p r=5$.

In the experiments performed in the year 2013 the initial segment (the first barrel) of the jet was transilluminated. Processing of the experimental data obtained at the VJU in 2013, according to the methods developed in [3], showed that the spectra are generally consistent with power law models $\Phi \sim \kappa^{-v}$ with index $v$ varying with the distance from the nozzle and the jet axis. It is seen that the index $v$ is much smaller than in the Kolmogorov-Obukhov model of developed turbulence (11/3) and gradually increases with the distance from the nozzle exit $h$. The most intense turbulence occurs in the mixing layer at the outer edge of the jet, as evidenced by faster growth of the parameter $v$ in this area compared to its values at the jet axis. Intense turbulence development leads to the increase of the index $v$ in this area relatively to its value near the axis (Table.1).

Table 1. Variations of the infex $v$ inside the first barrel.

\begin{tabular}{|c|c|c|c|c|}
\hline & $h=\mathbf{3} \mathbf{~ m m}$ & $\boldsymbol{h}=\mathbf{8} \mathbf{~ m m}$ & $h=\mathbf{1 8} \mathbf{~ m m}$ & $h=\mathbf{2 8 . 5} \mathbf{~ m m}$ \\
\hline $\boldsymbol{r}=\mathbf{0}$ & 0.45 & 1.1 & 1.3 & 1.65 \\
\hline $\boldsymbol{r}=\mathbf{1 0} \mathbf{~ m m}$ & 0.5 & 1.2 & 1.5 & 1.85 \\
\hline
\end{tabular}

Experimental data of the year 2016 refer to the next barrel of the jet (heights $h=42-90$ $\mathrm{mm}$ from the nozzle exit). Examples of spectral density of laser radiation passing through the jet at $h=42 \mathrm{~mm}$ from the nozzle for impact parameters in range $0-20 \mathrm{~mm}$ are shown on Figure 3. According to [4], the high frequency asymptotes have the power index $-v-1$. Analysis of the results of 2016 showed that the power model, in general, good fits the spectra in the interval of heights $42-90 \mathrm{~mm}$ from the nozzle exit also, however, the values of the index $v$ in the second barrel are noticeably superior to its value in the first barrel but are still less than for the developed atmospheric turbulence (11/3). The second barrel of the values of the index lie in the range 2-2.7 (Table. 2). The index $v$ appears to be minimal at the jumps of averaged parameters of the jet (internal and external mixing layer, Mach disk). Probably it is the consequence of the presence of spatially coherent sources of turbulence importing whirlwinds with relatively large spatial scales, which degradation into small whirlwinds happens in more distant jet area.

We have to note that the values of the index of the spectrum in the second barrel are close to the model of coherent turbulence [5], describing the decay of large eddies with scales close to the size of the turbulence source. The increase of the index with the increase 
of turbulence and deceleration of the jet corresponds to the transition to the subsonic flow regime [6].

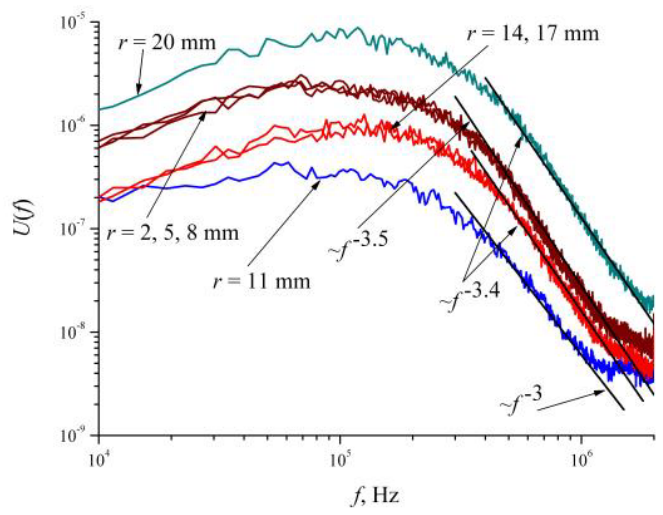

Fig. 3. Transformation of the laser radiation spectra with the increase of impact parameter $(h=42 \mathrm{~mm}, n p r=5)$.

Table 2. Variations of the infex $\mathrm{v}$ inside the second barrel.

\begin{tabular}{|c|c|c|c|c|c|}
\hline & $h=\mathbf{4 2} \mathbf{~ m m}$ & $h=\mathbf{5 0} \mathbf{~ m m}$ & $h=\mathbf{6 0} \mathbf{~ m m}$ & $h=\mathbf{7 0} \mathbf{~ m m}$ & $h=\mathbf{8 0} \mathbf{~ m m}$ \\
\hline $\boldsymbol{r = 0}$ & 2.3 & 2.0 & 1.9 & 2.0 & 2.4 \\
\hline $\boldsymbol{r = 7} \mathbf{~ m m}$ & 2.3 & 2.2 & 2.0 & 2.3 & 2.7 \\
\hline $\begin{array}{c}\boldsymbol{r}=\mathbf{1 3} \\
\mathbf{m m}\end{array}$ & 2.1 & 2.3 & 1.9 & 2.1 & 2.5 \\
\hline
\end{tabular}

\section{Conclusions}

Thus, the reconstruction of the spectra of the refractive index from the data of laser scanning shows that the development of turbulent processes in axially symmetric supersonic jet is described by a power-law model of the spectrum of refractive index with rate $v$ varying along the jet axis and its radius. The main changes correspond to the increase of the rate as the turbulence of the flow. The result is a small increase in index from the axis to the outer boundary of the jet and its noticeable increase along the axis in the first and second barrels. Small variations appear near the sources of turbulence (Mach disk, mixing layer). Values of power index correspond to the transformation of the coherent turbulence model to the developed turbulence one.

This work was supported by the RFBR grant №15-08-06549 A.

\section{References}

1. N.A. Fomin, Speckle Photography for Fluid Mechanics Measurements. Series: Experimental Fluid Mechanics. (Springer-Verlag, Berlin, 1998)

2. G. Meier, Exp. Fluids, 33, 181 (2002)

3. D.A. Marakasov, Proc. SPIE 10035, 100352B-1 (2016)

4. D.A. Marakasov, V.M. Sazanovich, A.A. Sukharev, R.Sh. Tsvyk, Atmospheric and Oceanic Optics 26, 11 (2013) 
5. V.V. Nosov, P.G. Kovadlo, V.P. Lukin, A.V. Torgaev, Atmospheric and Oceanic Optics 26, 201 (2013)

6. I.A. Joia, B.J. Uscinski, , R.J. Perkins, G. Balmer, , D. Jordan, E. Jakeman, Waves in Random Media 7, 169 (1997) 